\title{
Bifurcation analysis of a forced delay equation for machine tool vibrations
}

\author{
János Lelkes $\mathbb{D}$ • Tamás Kalmár-Nagy
}

Received: 15 December 2018 / Accepted: 25 April 2019 / Published online: 6 May 2019

(C) The Author(s) 2019

\begin{abstract}
A machining tool can be subject to different kinds of excitations. The forcing may have external sources (such as rotating imbalance, misalignment of the workpiece or ultrasonic excitation), or it can arise from the cutting process itself (e.g., periodic chip formation). We investigate the classical one-degreeof-freedom tool vibration model, a delay-differential equation with quadratic and cubic nonlinearity, and periodic forcing. The method of multiple scales is used to derive the slow flow equations. Stability and bifurcation analysis of equilibria of the slow flow equations is presented. Analytical expressions are obtained for the saddle-node and Hopf bifurcation points. Bifurcation analysis is also carried out numerically. Sub- and supercritical Hopf, cusp, fold, generalized Hopf (Bautin), Bogdanov-Takens bifurcations are found. Limit cycle continuation is performed using MatCont. Local and global bifurcations are studied and illustrated with phase portraits and direct numerical integration of the original equation.
\end{abstract}

Keywords Time delay $\cdot$ Retarded systems $\cdot$ Multiple scales $\cdot$ Bifurcation $\cdot$ MatCont

J. Lelkes $(\varangle) \cdot$ T. Kalmár-Nagy

Department of Fluid Mechanics, Faculty of Mechanical

Engineering, Budapest University of Technology and

Economics, Budapest, Hungary

e-mail: jani1234321@gmail.com

T. Kalmár-Nagy

e-mail: nody@kalmarnagy.com

\section{Introduction}

Delay-differential equations appear in many areas of engineering and science. For example in automotive engineering, manufacturing, neuroscience, population dynamics, economics, and control theory (see [1-4]).

Various techniques like the method of multiple scales [5-10], the Linstedt-Poincaré method [11], the combination of the method of multiple scales and the Linstedt-Poincaré method [12], harmonic balance method [13-15], method of averaging [16], semidiscretization [17], center manifold reduction [18,19] and normal forms [20] have been used to study delaydifferential equations.

Our focus here is harmonically forced delay-differential equations. These type of equations have been studied in the past decades [21-31]. In particular, we will study machine tool vibrations with external forcing. Tool vibrations are described by delay-differential equations, since the chip thickness, which determines the cutting force, is affected both by actual and delayed tool positions due to the surface regeneration effect [32-37].

To better understand the machining process, the chip formation has to be modeled. Periodic chip formation was investigated in [38-46]. In recent works of Csernák and Pálmai [47,48], a nonlinear system of differential equations is used to model chip segmentation. They find periodic, aperiodic and chaotic behavior of the chip 
formation model, which provides an excitation within the machine-tool-workpiece system.

Vibration-assisted machining is another example of externally excited machine tool vibrations, where the small-amplitude, high-frequency tool displacement leads to improved surface finish and accuracy compared to conventional machining [49-52].

\section{The model and its linear stability analysis}

In this paper, a harmonically excited single-degree-offreedom machine tool vibration model of orthogonal cutting (Fig. 1) is investigated (for derivation see [14, 18])

$$
\begin{aligned}
\ddot{x}+2 \zeta \dot{x}+x= & -p\left(x-x_{\tau}\right) \\
& +q\left(\left(x-x_{\tau}\right)^{2}-\left(x-x_{\tau}\right)^{3}\right) \\
& +A \cos (\omega t),
\end{aligned}
$$

where $x$ is the tool displacement, $\zeta>0$ is the relative damping factor, $p>0$ is the nondimensional cutting force, $q>0$ is the coefficient of nonlinearity, $A \geq 0$ is the amplitude of the forcing, and $\omega$ is its frequency. The term $x_{\tau}$ denotes $x(t-\tau)$, the delayed value of the position with with positive delay $\tau$. The regenerative effect is considered with the expressions containing the dimensionless chip thickness variation $x-x_{\tau}$ on the right-hand side of Eq. (1).

The stability analysis of the $x=0$ solution of the linearized equation

$\ddot{x}+2 \zeta \dot{x}+x=-p\left(x-x_{\tau}\right)$,

was performed in $[36,53]$. The characteristic function of Eq. (2) is obtained by substituting the trial solution $x(t)=C \exp (i \Omega t)$ into Eq. (2). The stability diagram in Fig. 2 is given in parametric form (see [14])

$$
\begin{aligned}
p & =\frac{\left(1-\Omega^{2}\right)^{2}+4 \zeta^{2} \Omega^{2}}{2\left(\Omega^{2}-1\right)}, \\
\tau & =\frac{2}{\Omega}\left(j \pi-\arctan \frac{\Omega^{2}-1}{2 \zeta \Omega}\right), \quad j=1,2, \ldots,
\end{aligned}
$$

where $j$ corresponds to the $j$ th 'lobe' and $\Omega>1$. At the minima ('notches') of the stability lobes, $\Omega, p, \tau$ assume the particularly simple forms

$\Omega_{\text {crit }}=\sqrt{1+2 \zeta}$

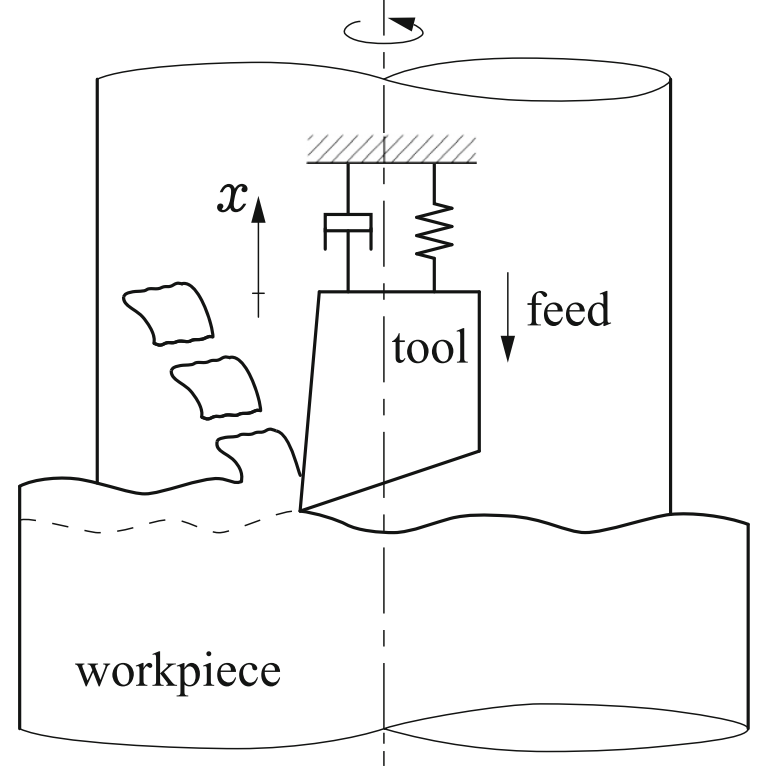

Fig. 1 One-degree-of-freedom mechanical model of orthogonal cutting [19]

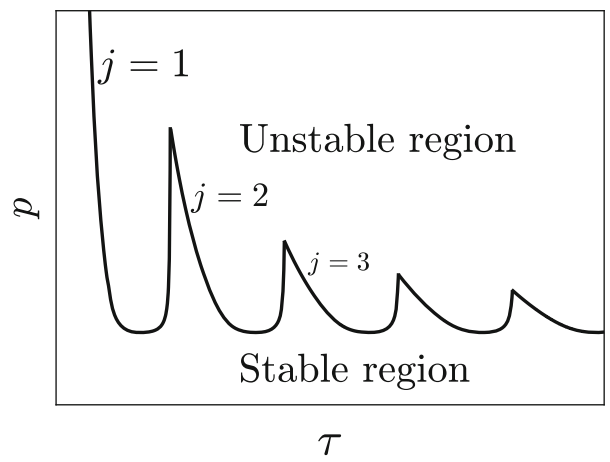

Fig. 2 The linear stability chart of Eq. (1)

$$
\begin{aligned}
p_{\text {crit }} & =2 \zeta(\zeta+1), \\
\tau_{\text {crit }} & =\frac{2\left(j \pi-\arctan \frac{1}{\sqrt{1+2 \zeta}}\right)}{\sqrt{1+2 \zeta}}, \quad j=1,2, \ldots
\end{aligned}
$$

Along the stability boundaries, Hopf bifurcation may occur, giving rise to periodic solutions of the nonlinear retarded system $[18,36]$.

\section{Slow flow equations and equilibria}

We approximate the solution of Eq. (1) by using the method of multiple scales. In the following we will assume that damping is small, nonlinearity and forcing 
are weak (see [14]) and the forcing is near-resonant, i.e.,

$\zeta, p, q, A \sim O(\varepsilon), \quad \omega=1+\sigma, \quad \sigma \sim O(\varepsilon)$.

Here $\varepsilon \ll 1$ is the bookkeeping parameter and $\sigma$ is the detuning frequency. We express the system parameters as

$$
\begin{aligned}
& \sigma=\varepsilon \hat{\sigma}, \quad \omega=1+\varepsilon \hat{\sigma}, \\
& p=\varepsilon \hat{p}, \quad q=\varepsilon \hat{q}, \quad \zeta=\varepsilon \hat{\zeta}, \quad A=\varepsilon \hat{A} .
\end{aligned}
$$

Substituting these into Eq. (1) we get

$$
\begin{aligned}
\ddot{x}+2 \varepsilon \hat{\zeta} \dot{x}+x= & -\varepsilon \hat{p}\left(x-x_{\tau}\right) \\
& +\varepsilon \hat{q}\left(\left(x-x_{\tau}\right)^{2}-\left(x-x_{\tau}\right)^{3}\right) \\
& +\varepsilon \hat{A} \cos ((1+\varepsilon \hat{\sigma}) t) .
\end{aligned}
$$

We also assume that the solution of Eq. (10) can be well approximated by the two-scale expansion

$x(t)=x_{0}\left(t_{0}, t_{1}\right)+\varepsilon x_{1}\left(t_{0}, t_{1}\right)+O\left(\varepsilon^{2}\right)$,

where the fast and slow timescales are defined as

$t_{0}=t, \quad t_{1}=\varepsilon t$

With the differential operators

$D_{0}=\frac{\partial}{\partial t_{0}}, \quad D_{1}=\frac{\partial}{\partial t_{1}}$,

time differentiation can be written as

$$
\frac{\mathrm{d}}{\mathrm{d} t}=D_{0}+\varepsilon D_{1}+O\left(\varepsilon^{2}\right)
$$

and second derivative with respect to time is

$$
\frac{\mathrm{d}^{2}}{\mathrm{~d} t^{2}}=D_{0}^{2}+2 \varepsilon D_{0} D_{1}+O\left(\varepsilon^{2}\right)
$$

Substituting the differential operators (14) and (15) into Eq. (10) and equating like powers of $\varepsilon$ one obtains

$\varepsilon^{0}: D_{0}^{2} x_{0}\left(t_{0}, t_{1}\right)+x_{0}\left(t_{0}, t_{1}\right)=0$,

$$
\begin{aligned}
\varepsilon^{1}: D_{0}^{2} x_{1} & \left(t_{0}, t_{1}\right)+x_{1}\left(t_{0}, t_{1}\right) \\
= & -2 D_{0} D_{1} x_{0}\left(t_{0}, t_{1}\right)-2 \hat{\zeta} D_{0} x_{0}\left(t_{0}, t_{1}\right) \\
& -\hat{p}\left[x_{0}\left(t_{0}, t_{1}\right)-x_{0}\left(t_{0}-\tau, t_{1}\right)\right] \\
& +\hat{q}\left[x_{0}\left(t_{0}, t_{1}\right)-x_{0}\left(t_{0}-\tau, t_{1}\right)\right]^{2} \\
& -\hat{q}\left[x_{0}\left(t_{0}, t_{1}\right)-x_{0}\left(t_{0}-\tau, t_{1}\right)\right]^{3} \\
& +\hat{A} \cos \left(t_{0}+\hat{\sigma} t_{1}\right) .
\end{aligned}
$$

Solving Eq. (16) for $x_{0}\left(t_{0}, t_{1}\right)$ yields

$x_{0}\left(t_{0}, t_{1}\right)=\alpha\left(t_{1}\right) \cos \left(t_{0}+\beta\left(t_{1}\right)\right)$,

where $\alpha\left(t_{1}\right)$ and $\beta\left(t_{1}\right)$ are the slowly varying and amplitude and phase, respectively. We now substitute this solution into Eq. (17). To eliminate the secular terms (terms containing $\sin \left(t_{0}+\beta\left(t_{1}\right)\right)$ and $\cos \left(t_{0}+\beta\left(t_{1}\right)\right)$ ), we require the following equations to hold:

$$
\begin{aligned}
D_{1} \alpha= & -\left(\hat{\zeta}+\frac{\hat{p}}{2} \sin \tau\right) \alpha \\
& -3 \hat{q} \cos \frac{\tau}{2} \sin ^{3} \frac{\tau}{2} \alpha^{3}+\frac{\hat{A}}{2} \sin \phi, \\
\alpha D_{1} \phi= & \frac{1}{2}(\hat{p}(\cos \tau-1)+2 \hat{\sigma}) \alpha \\
& -3 \hat{q} \sin ^{4} \frac{\tau}{2} \alpha^{3}+\frac{\hat{A}}{2} \cos \phi,
\end{aligned}
$$

where we introduced

$\phi\left(t_{1}\right)=\hat{\sigma} t_{1}-\beta\left(t_{1}\right)$.

Equations $(19,20)$ are two ordinary differential equations describing the evolution of the amplitude and phase (slow flow equation). We note that Eq. (20) becomes an algebraic equation for $\alpha=0$; therefore, we only consider the $\alpha>0$ case.

Without the secular terms, the integration of Eq. (17) gives

$$
\begin{aligned}
x_{1}\left(t_{0}, t_{1}\right)= & \hat{g}_{0} \alpha^{2}\left(t_{1}\right)+\hat{g}_{1} \alpha^{2}\left(t_{1}\right) \cos \left(2\left(t_{0}+\beta\left(t_{1}\right)\right)\right) \\
& +\hat{g}_{2} \alpha^{2}\left(t_{1}\right) \sin \left(2\left(t_{0}+\beta\left(t_{1}\right)\right)\right) \\
& +\hat{g}_{3} \alpha^{3}\left(t_{1}\right) \cos \left(3\left(t_{0}+\beta\left(t_{1}\right)\right)\right) \\
& +\hat{g}_{4} \alpha^{3}\left(t_{1}\right) \sin \left(3\left(t_{0}+\beta\left(t_{1}\right)\right)\right)
\end{aligned}
$$


where

$$
\begin{aligned}
& \hat{g}_{0}=\frac{\hat{q}(1-\cos (\tau))}{4}, \\
& \hat{g}_{1}=\frac{\hat{q}(2 \cos (\tau)-\cos (2 \tau)-1)}{6}, \\
& \hat{g}_{2}=\frac{\hat{q}(2 \cos (\tau)-\cos (2 \tau))}{6}, \\
& \hat{g}_{3}=\frac{\hat{q}(1-3 \cos (\tau)+3 \cos (2 \tau)-\cos (3 \tau))}{32}, \\
& \hat{g}_{4}=\frac{\hat{q}(-3 \cos (\tau)+3 \cos (2 \tau)-\cos (3 \tau))}{32} .
\end{aligned}
$$

Substituting Eqs. (18) and (22) into Eq. (11) and using Eq. (21), we get the multiple scales solution

$$
\begin{aligned}
x(t) \approx & x_{0}\left(t_{0}, t_{1}\right)+\varepsilon x_{1}\left(t_{0}, t_{1}\right) \\
= & \alpha\left(t_{1}\right) \cos \left(\omega t_{0}-\phi\left(t_{1}\right)\right)+g_{0} \alpha^{2}\left(t_{1}\right) \\
& +g_{1} \alpha^{2}\left(t_{1}\right) \cos \left(2\left(\omega t_{0}-\phi\left(t_{1}\right)\right)\right) \\
& +g_{2} \alpha^{2}\left(t_{1}\right) \sin \left(2\left(\omega t_{0}-\phi\left(t_{1}\right)\right)\right) \\
& +g_{3} \alpha^{3}\left(t_{1}\right) \cos \left(3\left(\omega t_{0}-\phi\left(t_{1}\right)\right)\right) \\
& +g_{4} \alpha^{3}\left(t_{1}\right) \sin \left(3\left(\omega t_{0}-\phi\left(t_{1}\right)\right)\right),
\end{aligned}
$$

where $g_{i}=\varepsilon \hat{g}_{i}, i \in 0,1,2,3,4$.

\subsection{Amplitude of the steady-state vibration}

The fixed points of the slow flow correspond to periodic solutions of the original Eq. (1). To get the amplitude $\alpha^{*}$ and the phase $\phi^{*}$ of the steady-state vibration, i.e., the fixed points of the slow flow equations $(19,20)$, we set the left-hand sides of Eqs. $(19,20)$ to zero, and multiply the expressions by $\varepsilon$, to get back the original $\zeta, p, q, A, \omega$ system parameters [see Eq. (9)]

$$
\begin{aligned}
& -\left(\zeta+\frac{p}{2} \sin \tau\right) \alpha^{*}-3 q \cos \frac{\tau}{2} \sin ^{3} \frac{\tau}{2} \alpha^{* 3} \\
& +\frac{A}{2} \sin \phi^{*}=0, \\
& \frac{1}{2}(p(\cos \tau-1)+2 \sigma) \alpha^{*}-3 q \sin ^{4} \frac{\tau}{2} \alpha^{* 3} \\
& +\frac{A}{2} \cos \phi^{*}=0 .
\end{aligned}
$$

We substitute $\sigma=(\omega-1)$ and solve the resulting algebraic equations (by eliminating the trigonometric terms from them). From these for the amplitude $\alpha^{*}$ and for the phase $\phi^{*}$, we get

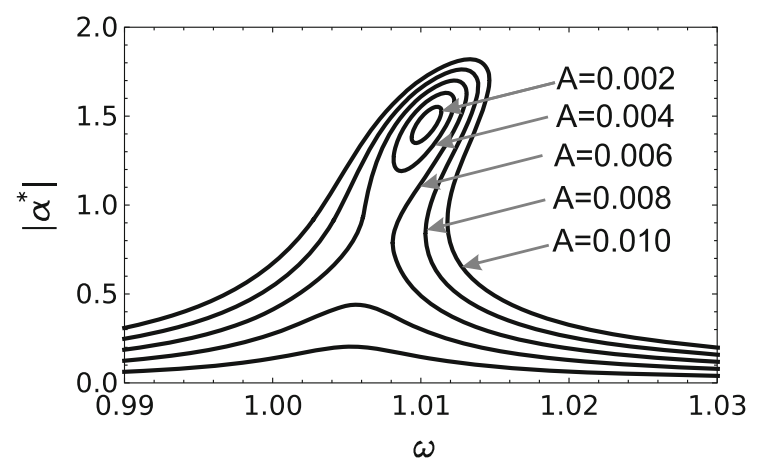

Fig. 3 The fixed point $\alpha^{*}$ as a function of $\omega$ for $A=$ $\{0.002,0.004,0.006,0.008,0.010\}$. The other parameters are $\tau=4.676, \xi=0.01, p=0.0101, q=0.003$

$$
\begin{aligned}
& {\left[\frac{\left(p(1-\cos \tau)-2(\omega-1)+6 q \sin ^{4} \frac{\tau}{2} \alpha^{* 2}\right)^{2}}{4}\right.} \\
& \left.\quad+\left(\zeta+\frac{p \sin \tau}{2}+3 q \cos \frac{\tau}{2} \sin ^{3} \frac{\tau}{2} \alpha^{* 2}\right)^{2}\right] \alpha^{* 2} \\
& \quad-\frac{A^{2}}{4}=0, \\
& \phi^{*}=\arcsin \left[\frac { 2 } { A } \left(\left(\zeta+\frac{p}{2} \sin \tau\right) \alpha^{*}\right.\right. \\
& \left.\left.\quad+3 q \cos \frac{\tau}{2} \sin ^{3} \frac{\tau}{2} \alpha^{* 3}\right)\right] .
\end{aligned}
$$

Note that Eq. (27) is an implicit function of the equilibrium amplitude $\alpha^{*}$. The shape of the equilibrium amplitude $\alpha^{*}$ as a function of $\omega$ is illustrated in Fig. 3 for different $A$ values.

\section{Analytical bifurcation analysis}

\subsection{Stability of the equilibria of the slow flow}

We examine the stability of the equilibrium points $\left(\alpha^{*}\right.$, $\left.\phi^{*}\right)$ of the slow flow Eqs. (19) and (20). Stability is determined by the eigenvalues

$\lambda_{1,2}=\frac{\operatorname{tr} \boldsymbol{J} \pm \sqrt{\operatorname{tr}^{2} \boldsymbol{J}-4 \operatorname{det} \boldsymbol{J}}}{2}$,

where $\boldsymbol{J}$ is the Jacobian

$$
\boldsymbol{J}=\left(\begin{array}{cc}
-\zeta-\frac{p}{2} \sin \tau-9 q \cos \frac{\tau}{2} \sin ^{3} \frac{\tau}{2} \alpha^{* 2} & \frac{A}{2} \cos \phi^{*} \\
-6 q \sin ^{4} \frac{\tau}{2} \alpha^{*}-\frac{A}{2 \alpha^{* 2}} \cos \phi^{*} & -\frac{A}{2 \alpha^{*}} \sin \phi^{*}
\end{array}\right) .
$$


Using Eqs. (25) and (26) we eliminate $\cos \phi^{*}$ and $\sin \phi^{*}$ from the Jacobian (30) to yield

$\boldsymbol{J}=\left(\begin{array}{ll}J_{11} & J_{12} \\ J_{21} & J_{22}\end{array}\right)$,

where

$$
\begin{aligned}
J_{11}= & -\left(\zeta+\frac{p}{2} \sin \tau\right)-9 q \cos \frac{\tau}{2} \sin ^{3} \frac{\tau}{2} \alpha^{* 2}, \\
J_{12}= & -\frac{1}{2}(p(\cos \tau-1)+2 \omega-2) \alpha^{*} \\
& +3 q \sin ^{4} \frac{\tau}{2} \alpha^{* 3}, \\
J_{21}= & \frac{1}{2}(p(\cos \tau-1)+2 \omega-2) \frac{1}{\alpha^{*}}-9 q \sin ^{4} \frac{\tau}{2} \alpha^{*}, \\
J_{22}= & -\left(\zeta+\frac{p}{2} \sin \tau\right)-3 q \cos \frac{\tau}{2} \sin ^{3} \frac{\tau}{2} \alpha^{* 2} .
\end{aligned}
$$

To determine the bifurcation points, the trace and the determinant of the Jacobian $\boldsymbol{J}$ will be useful

$$
\operatorname{tr} \boldsymbol{J}=-12 q \cos \frac{\tau}{2} \sin ^{3} \frac{\tau}{2} \alpha^{* 2}-2\left(\zeta+\frac{p}{2} \sin \tau\right),
$$

$\operatorname{det} \boldsymbol{J}=b_{2}\left(\alpha^{* 2}\right)^{2}+b_{1}(\omega)\left(\alpha^{* 2}\right)+b_{0}(\omega)$,

where

$$
\begin{aligned}
b_{2}= & 27 q^{2} \sin ^{6} \frac{\tau}{2}, \\
b_{1}(\omega) & =12 q \sin ^{3} \frac{\tau}{2}\left(\zeta \cos \frac{\tau}{2}+(p-\omega+1) \sin \frac{\tau}{2}\right), \\
b_{0}(\omega) & =\zeta^{2}+\frac{p^{2}}{2}-p \omega+p+(\omega-1)^{2}+\zeta p \sin \tau \\
& -\frac{1}{2} p(p-2 \omega+2) \cos \tau .
\end{aligned}
$$

We note that $\operatorname{tr} \boldsymbol{J}$ is a linear function and $\operatorname{det} \boldsymbol{J}$ is a second-order polynomial of $\alpha^{* 2}$.

\subsection{Saddle-node bifurcation}

A saddle-node bifurcation of an equilibria occurs when

$\operatorname{det} \boldsymbol{J}=b_{2}\left(\alpha^{* 2}\right)^{2}+b_{1}(\omega) \alpha^{* 2}+b_{0}(\omega)=0$.

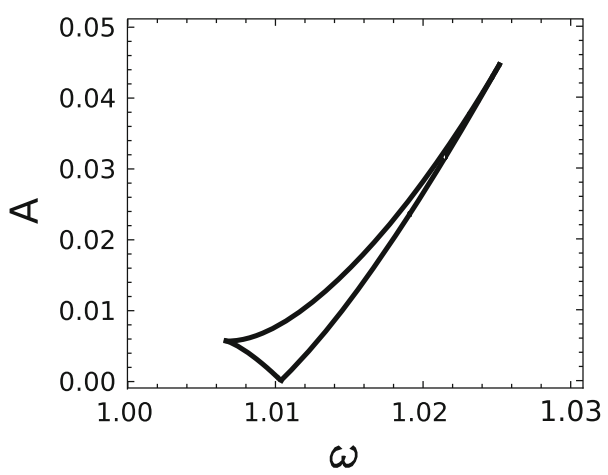

Fig. 4 Saddle-node curves on the $\omega-A$ plane for the parameters $\tau=4.676, \xi=0.01, p=0.0101, q=0.003$

Equation (27) implicitly determines equilibria and can be written as a third-order polynomial of $\alpha^{* 2}$

$$
\frac{b_{2}}{3}\left(\alpha^{* 2}\right)^{3}+\frac{b_{1}(\omega)}{2}\left(\alpha^{* 2}\right)^{2}+b_{0}(\omega) \alpha^{* 2}-\frac{A^{2}}{4}=0 .
$$

Eliminating $\alpha^{* 2}$ from the simultaneous Eqs. (36) and (37) yields

$$
\begin{gathered}
9 b_{2}^{2} A^{4}+\left(36 b_{0}(\omega) b_{1}(\omega) b_{2}-6 b_{1}^{3}(\omega)\right) A^{2} \\
+64 b_{0}^{3}(\omega) b_{2}-12 b_{0}^{2}(\omega) b_{1}^{2}(\omega)=0 .
\end{gathered}
$$

Provided $\frac{16}{3} b_{2} b_{0}(\omega) \geq b_{1}^{2}(\omega) \geq 4 b_{2} b_{0}(\omega)$, we get 2 values

$$
A=\sqrt{\frac{b_{1}^{3}(\omega)-6 b_{0}(\omega) b_{1}(\omega) b_{2} \pm\left(b_{1}^{2}-4 b_{0}(\omega) b_{2}\right)^{3 / 2}}{3 b_{2}^{2}}} .
$$

Equation (39) determines the two curves on the $\omega-A$ plane where saddle-node bifurcation occurs (Fig. 4).

\subsection{Hopf bifurcation}

Equation (37) implicitly determines equilibria. A Hopf bifurcation of equilibrium point can occur if $\operatorname{tr} \boldsymbol{J}=0$ and $\operatorname{det} \boldsymbol{J}>0$ (necessary conditions). To get the sufficient condition of the Hopf bifurcation, the transversality (positive root crossing velocity) and genericity (equilibrium is weakly attracting/repelling) conditions have to be fulfilled [54]. 
We start with

$\operatorname{tr} \boldsymbol{J}=-12 q \cos \frac{\tau}{2} \sin ^{3} \frac{\tau}{2} \alpha^{* 2}-2\left(\zeta+\frac{p}{2} \sin \tau\right)=0$

From Eq. (40) we express

$\alpha^{* 2}=-\frac{\zeta+\frac{p}{2} \sin \tau}{6 q \cos \frac{\tau}{2} \sin ^{3} \frac{\tau}{2}}$,

and substitute it into Eq. (37) to get an implicit function

$\mathcal{H}(\omega, A)=h_{2} \omega^{2}+h_{1} \omega+h_{0}-\frac{A^{2}}{4}=0$,

where

$$
\begin{aligned}
h_{2}= & -\frac{\zeta+\frac{p}{2} \sin \tau}{6 q \cos \frac{\tau}{2} \sin ^{3} \frac{\tau}{2}}\left(=\alpha^{* 2}\right), \\
h_{1}= & \frac{p^{2}+4 \csc \tau\left(\zeta \csc ^{2} \frac{\tau}{2}+\left(p-\zeta^{2}\right) \csc \tau+p \cot \tau\right)}{6 q} \\
h_{0}= & \frac{p^{2}(p+4) \cos 2 \tau-4 \zeta p^{2}\left(\sin \tau+\sin 2 \tau+32 \zeta^{2}\right)}{48 q \sin ^{2} \tau} \\
& -\frac{16 \zeta\left(\zeta^{2}+2 \cos \tau+2\right) \csc \tau+16\left(\zeta^{2}+1\right) p \cos \tau}{48 q \sin ^{2} \tau} \\
& +\frac{p^{2}+4 p-8 p\left(\zeta^{2}+2\right)}{48 q \sin ^{2} \tau} .
\end{aligned}
$$

We eliminate $\alpha^{* 2}$ from the $\operatorname{det} \boldsymbol{J}>0$ condition by substituting Eq. (41) into Eq. (34) to get the inequality as the function of $\omega$

$$
\begin{aligned}
\mathcal{D}(\omega)= & \omega^{2}+\left(2 \zeta \tan \frac{\tau}{2}-2\right) \omega+\frac{4 \zeta^{2}-p^{2}+8}{8(\cos \tau+1)} \\
& +\frac{\left(8-8 \zeta^{2}+p^{2} \cos \tau\right) \cos \tau-4 \zeta(p+4) \sin \tau}{8(\cos \tau+1)}>0 .
\end{aligned}
$$

Equation (42) together with the inequality (44) determines the possible Hopf bifurcation points in the $(\omega, A)$ plane. For given parameter values such a figure is shown in Fig. 12.

When $\mathcal{H}(\omega, A)=0$ and $\mathcal{D}(\omega)=0$ we observe Bogdanov-Takens bifurcation points on the Hopf curve.

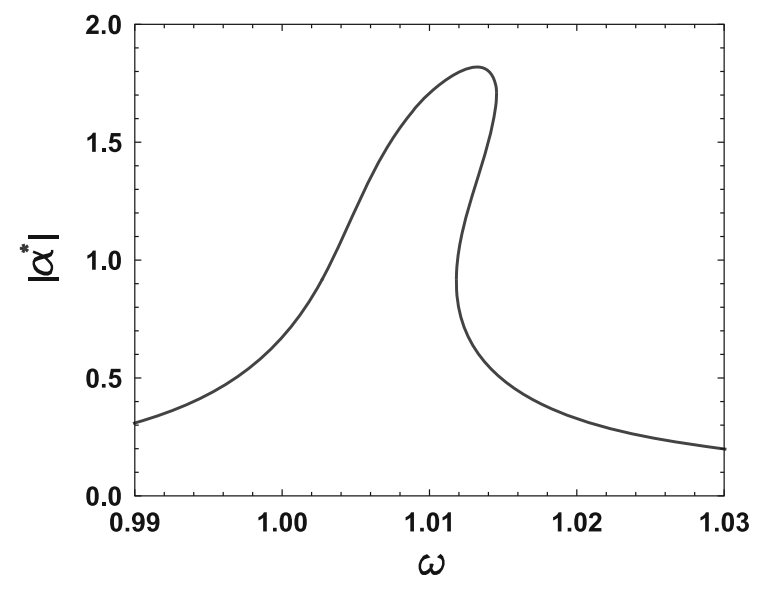

Fig. 5 The equilibrium amplitude as the function of $\omega$

\section{Numerical results of the bifurcation analysis}

The angular frequency $\omega$ of the forcing was chosen as a bifurcation parameter. As in $[18,55,56]$ we set the other parameter values at the first lobe ( $j=1$, see Fig. 2) and weak nonlinearity and forcing

$$
\begin{aligned}
& \tau=4.676, \quad \zeta=0.01, \quad p=0.5 p_{\text {crit }}=0.0101, \\
& q=0.003, \quad A=0.01 .
\end{aligned}
$$

The equilibrium amplitude $\alpha^{*}$ as a function of $\omega$ is illustrated in Fig. 5 (this is the uppermost curve of Fig. 3). Using Eq. (39) saddle-node bifurcations occur at

$\omega_{S N 1}=1.0118, \quad \omega_{S N 2}=1.0146$.

While at $\omega_{S N 1}$ a regular saddle-node bifurcation occurs, at $\omega_{S N 2}$ a so-called homoclinic saddle-node bifurcation [57] can be observed. At this point a limit cycle is born (see Fig. 8f, g).

Using Eqs. (42) and inequality (44), Hopf bifurcation occurs at

$\omega_{\mathrm{Hopf}}=1.00363$.

At this point the stable equilibrium point becomes unstable and a subcritical Hopf bifurcation occurs.

The bifurcation diagram is shown in Fig. 6. 


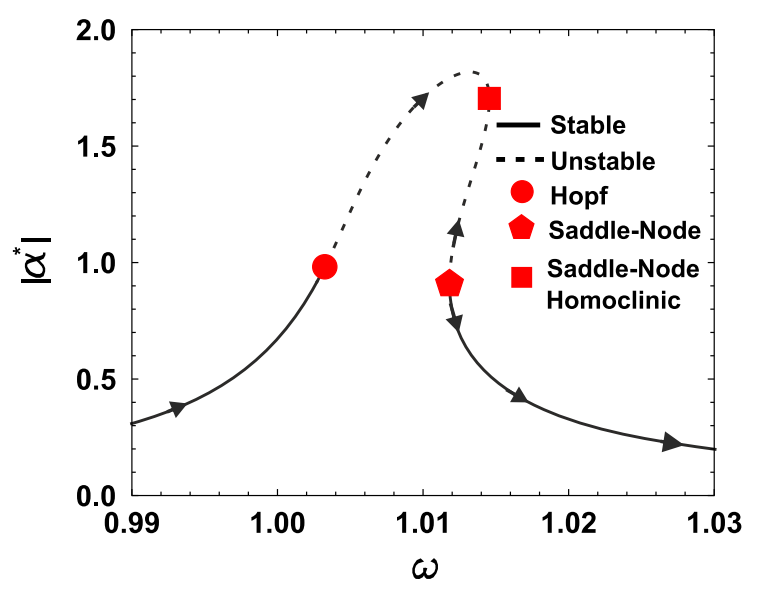

Fig. 6 Bifurcation diagram

\subsection{Phase plane and phase portraits}

To illustrate the dynamical behavior of the system, we chose the following angular frequencies of the forcing

$$
\begin{array}{ll}
\omega_{I}=1, & \omega_{I I}=\omega_{\mathrm{Hopf}}=1.00363 \\
\omega_{I I I}=1.005, & \omega_{I V}=\omega_{S N 1}=1.0118 \\
\omega_{V}=1.013, & \omega_{V I}=\omega_{S N 2}=1.0146 \\
\omega_{V I I}=1.02 . &
\end{array}
$$

The right-hand sides of Eqs. (19) and (20) are periodic functions of the phase $\phi$ with period $2 \pi$, thus $(\alpha, \phi) \in$ $\left(\mathbb{R}^{1}, S^{1}\right)$.The true phase space is a cylindrical surface, see Fig. 7.

Figure 8 shows the phase portrait of the slow flow at various forcing frequencies. The filled circles denote the stable, the empty circles the unstable equilibria and the dashed lines correspond to the unstable limit cycles.

At $\omega=\omega_{I}$ the equilibrium (filled circle) is a stable spiral and the thick dashed line is a $2 \pi$-periodic unstable limit cycle (Fig. 8a). At $\omega=\omega_{I I}$ a pair of complex conjugate eigenvalues cross the imaginary axis. At this point the fixed point is weakly repelling, giving rise to a subcritical Hopf bifurcation (Fig. 8b). After the Hopf bifurcation point $\left(\omega=\omega_{I I I}\right)$ the stable equilibrium becomes unstable (Fig. 8c) and all solutions go off to infinity. Further increasing the bifurcation parameter $\omega$ the unstable spiral equilibrium (empty circle) becomes an unstable node. In Fig. $8 \mathrm{~d}$ at $\omega=\omega_{I V}$ the left dot $\left(\alpha^{*}=1.72\right)$ is the unstable node, the right dot $\left(\alpha^{*}=0.93\right)$ is a non-hyperbolic fixed point undergoing saddle-node bifurcation. After the saddle-

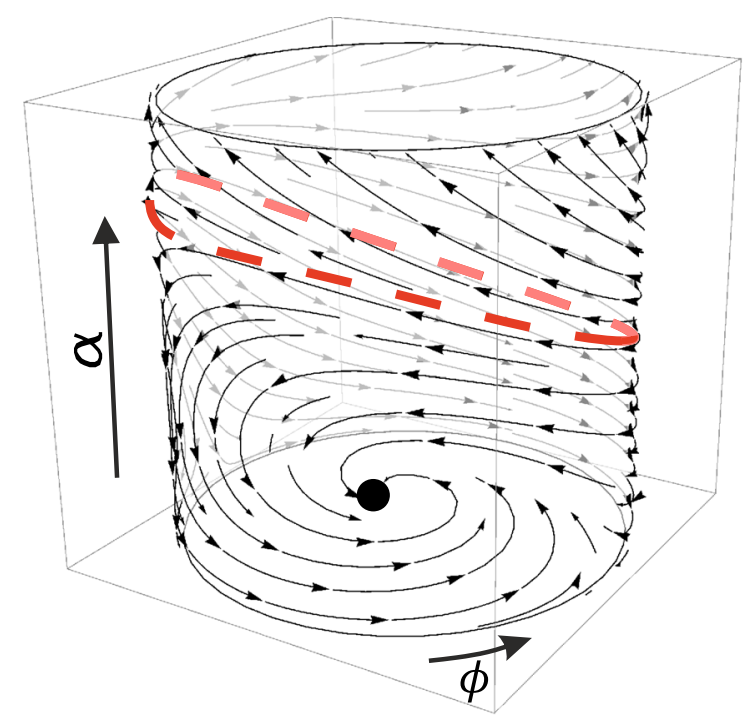

Fig. 7 Phase cylinder at $\omega=\omega_{I}$. Dashed line-unstable limit cycle, filled circle—stable equilibrium

node bifurcation a stable node and a saddle point is created (Fig. 8e at $\omega=\omega_{V}$ ). The stable node transforms into a stable spiral at $\omega=1.0119>\omega_{V}$ and the saddle point moves toward the unstable node. At $\omega=\omega_{V I}$ the unstable node and saddle point coalesce and a saddle-node homoclinic bifurcation (global bifurcation) occurs (Fig. 8f). After the saddle-node homoclinic bifurcation $\left(\omega=\omega_{V I I}\right)$ an unstable limit cycle (thick arrowless line) is created (see Fig. 8g).

\subsection{Global bifurcations}

At $\omega=1.00327<\omega_{\text {Hopf }}$ the unstable limit cycle around the equilibrium point "collides" with the nonadmissible line $\alpha=0$ giving rise to a "global" $(2 \pi$ periodic in $\phi$ ) unstable limit cycle (Fig. 9).

Another global bifurcation is a homoclinic saddlenode bifurcation [57]. This occurs at $\omega=\omega_{V I}$. The coalescence of the saddle and unstable node is a homoclinic bifurcation and a "global" unstable limit cycle is born (see Fig. 10).

\subsection{The $\omega-A$ plane}

Now we consider the forcing frequency $\omega$ and amplitude $A$ as two bifurcation parameters. The other system 


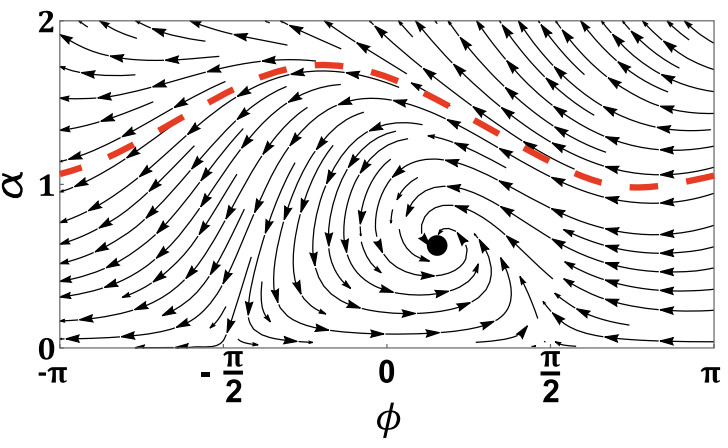

(a) $\omega=\omega_{I}$

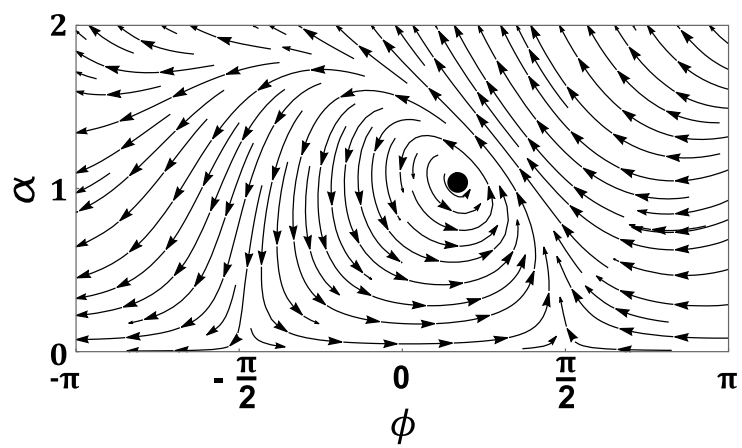

(b) $\omega=\omega_{I I}$

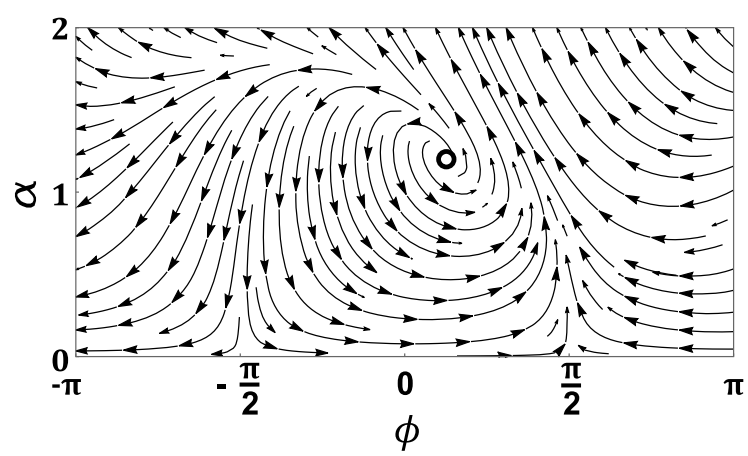

(c) $\omega=\omega_{I I I}$

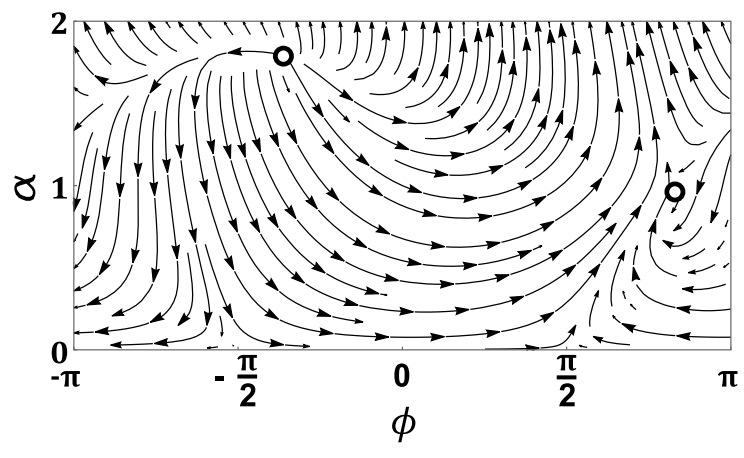

(d) $\omega=\omega_{I V}$

Fig. 8 Phase portrait at various forcing frequencies

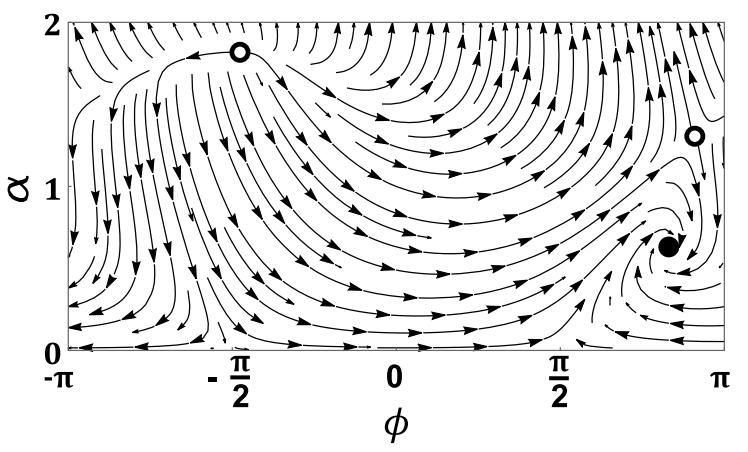

(e) $\omega=\omega_{V}$

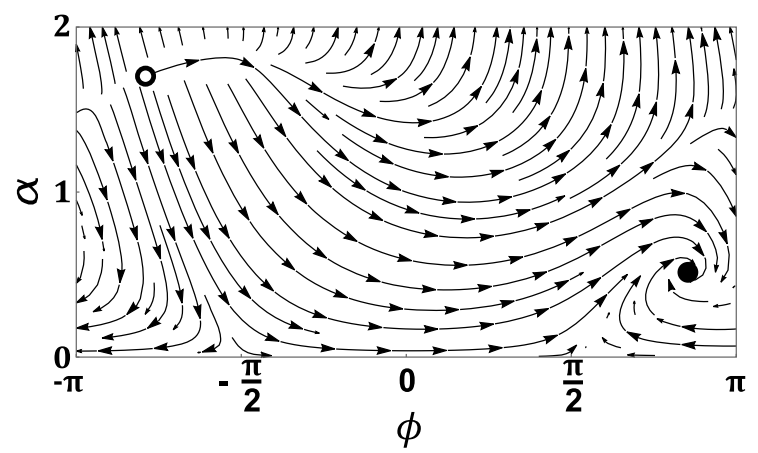

(f) $\omega=\omega_{V I}$

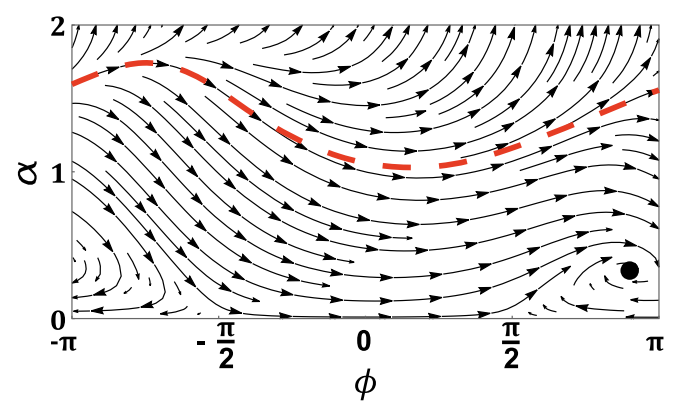

(g) $\omega=\omega_{V I I}$

Fig. 8 continued

parameters were the same as in Eq. (45). We determined the saddle-node curves together with the cusp and Bogdanov-Takens points with MatCont [58-61] (see Fig. 11). The boundary of the gray region is the same as the analytical result (39) (see also Fig. 4).

Inside the closed wedge (filled with gray in Fig. 11) three equilibrium points of the slow flow exist, two on the boundary and one outside the wedge. At the corners of the wedge we have three cusp (CP) bifurcation points. Equations (42) and (44) determine two Bogdanov-Takens (BT) points [54]. Figure 12 shows the Hopf bifurcation curve determined by Eq. (42). 


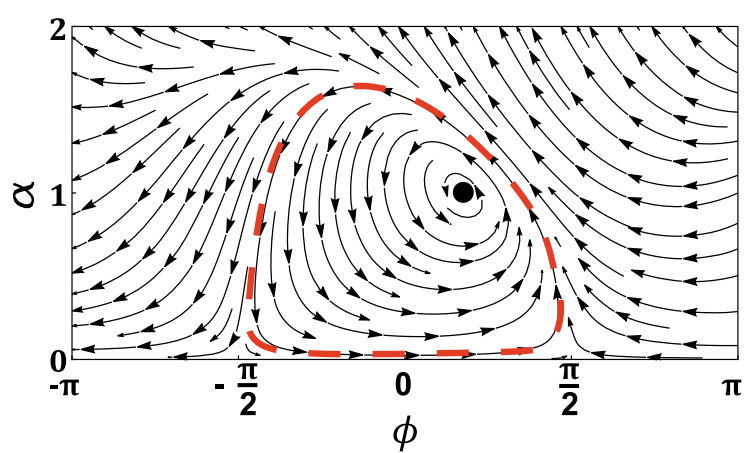

(a)

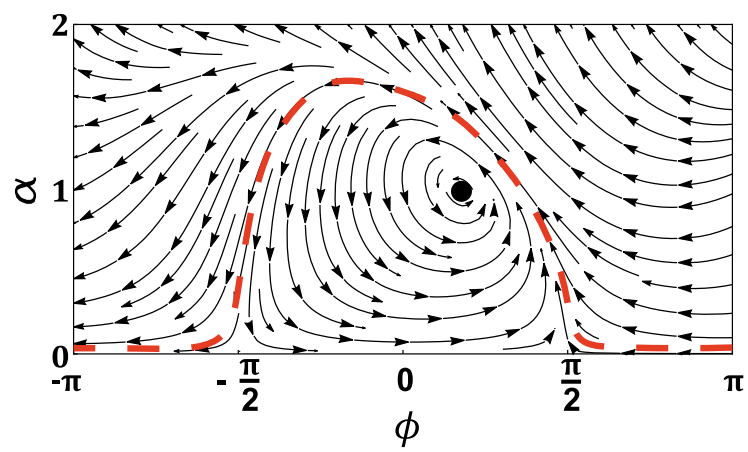

(b)

Fig. 9 Trajectories before and after the global bifurcation

Using MatCont we determined the Generalized Hopf (Bautin bifurcation) point. At the Generalized Hopf bifurcation point, the first Lyapunov coefficient vanishes [54]. This bifurcation point separates branches of subcritical and supercritical Hopf bifurcations (left segment until GH and GH-BT segment in Fig. 12). The segment BT-BT corresponds to neutral saddle points. In a neighborhood of the GH point, fold (saddle-node) bifurcation of limit cycles occurs. To illustrate this, we chose 4 points (denoted by squared in Fig. 13), one before and three after the Generalized Hopf bifurcation point.

To trace the limit cycles we used MatCont, with the bifurcation parameter $\omega$. At the first point $A=$ 0.0075 the Hopf bifurcation is subcritical (Fig. 14a). After the Generalized Hopf bifurcation point the Hopf bifurcation becomes supercritical. At $A=0.0070$ and $A=0.0065$ the system has two limit cycles which collide and disappear in a fold bifurcation (Fig. 14b, c).

The two coexisting limit cycles (small limit cyclestable, large limit cycle—unstable) at $\omega=1.00587$,

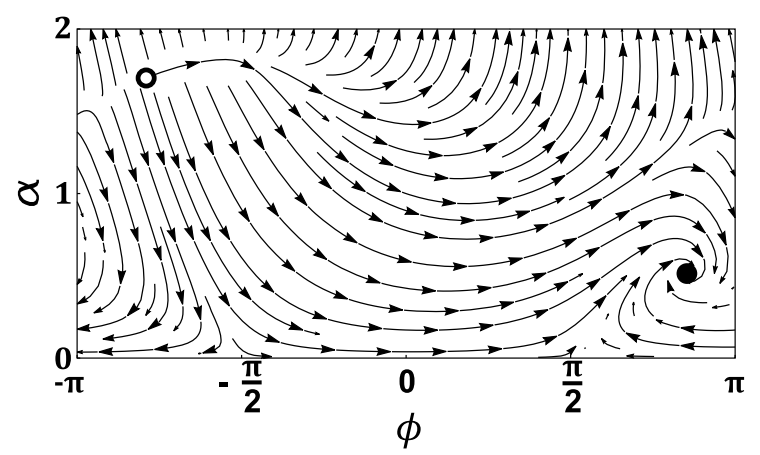

(a)

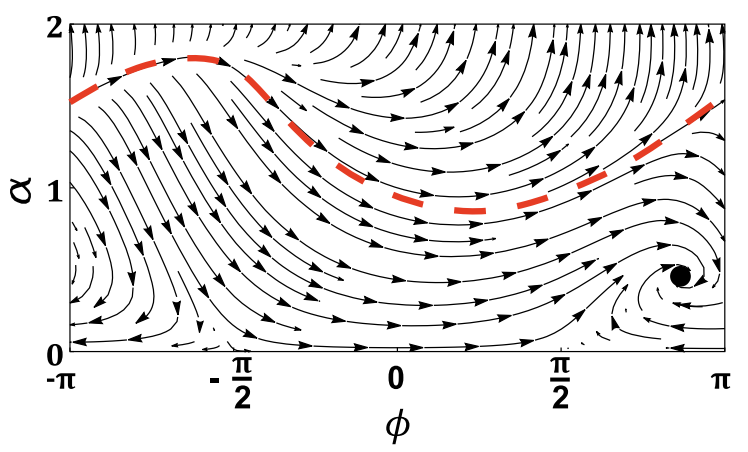

(b)

Fig. 10 Trajectories before and after the homoclinic saddle-node bifurcation

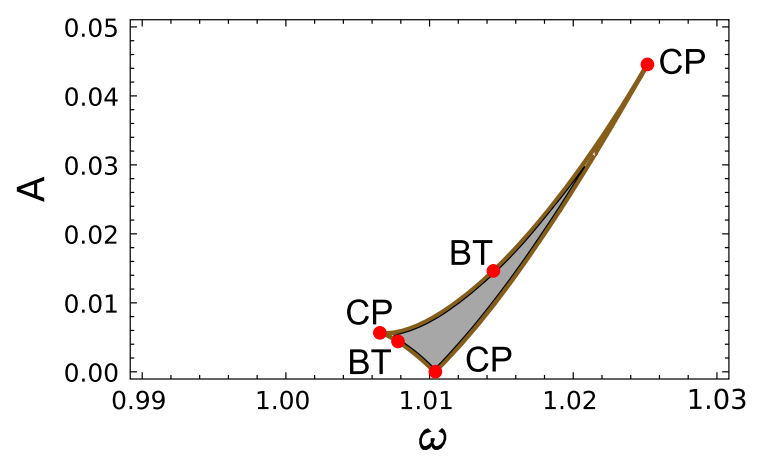

Fig. 11 Bifurcation diagram ( $C P$ cusp, $B T$ Bogdanov-Takens)

$A=0.0065$ are illustrated in Fig. 15. At $A=0.0060$ the system has one limit cycle again (Fig. 14d).

\section{Comparison of the method of multiple scales and direct numerical solution}

The direct numerical solution of Eq. (1) with parameters specified in Eq. (45) was determined in 


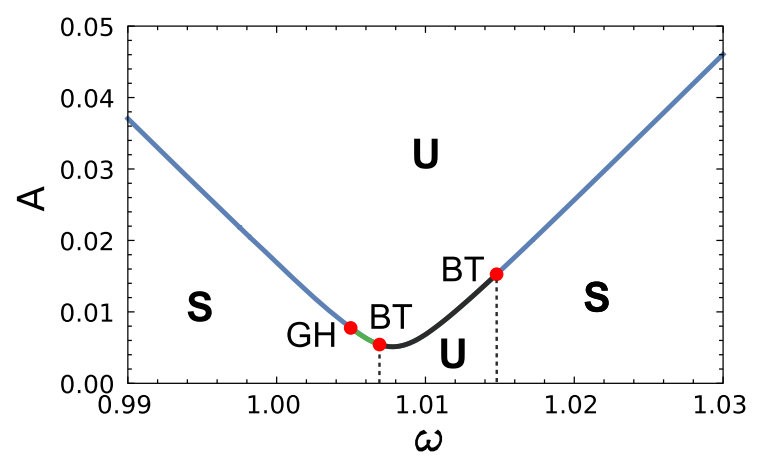

Fig. 12 Bifurcation diagram (BT Bogdanov-Takens, $G H$ Generalized Hopf, $\mathbf{S}$ stable region, $\mathbf{U}$ unstable region)

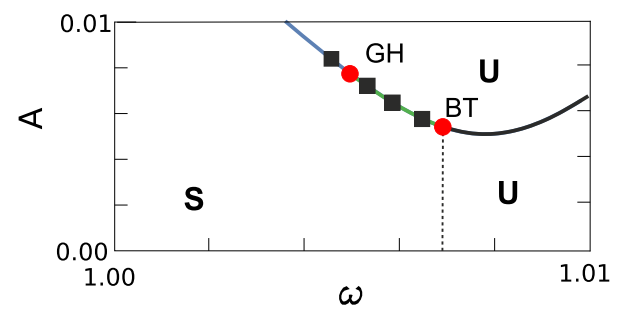

Fig. 13 Bifurcation diagram (BT Bogdanov-Takens, $G H$ Generalized Hopf, $\mathbf{S}$ stable region, $\mathbf{U}$ unstable region). The squares denote the starting points of the limit cycle continuation

Mathematica using the built in adaptive Runge-Kutta method. The numerical simulation is started with the initial function

$$
\begin{aligned}
x(t)= & \alpha^{*} \cos \left(\omega t-\phi^{*}\right)+g_{0} \alpha^{* 2} \\
& +g_{1} \alpha^{* 2} \cos \left(2\left(\omega t-\phi^{*}\right)\right) \\
& +g_{2} \alpha^{* 2} \sin \left(2\left(\omega t-\phi^{*}\right)\right) \\
& +g_{3} \alpha^{* 3} \cos \left(3\left(\omega t-\phi^{*}\right)\right) \\
& +g_{4} \alpha^{* 3} \sin \left(3\left(\omega t-\phi^{*}\right)\right), \quad t \in[-\tau, 0] .
\end{aligned}
$$

where $\alpha^{*}$ and $\phi^{*}$ were determined numerically from Eqs. (27) and (28).

Figure 16 illustrates the direct numerical solution (NUM) of Eq. (1) and the slow flow (SF) solution Eqs. (19) and (20). The fixed points of the slow flow equations (constant amplitude $x(t)$ in Fig. 16a) are the periodic solution of the original delay equation. Figure $16 \mathrm{~b}$ shows that the slow flow equations capture the amplitude dynamics even away from an equilibrium point.

The unstable limit cycles of the subcritical Hopf bifurcations of the slow flow equations $(19,20)$ are unstable quasi-periodic solutions of Eq. (1). Figure 17

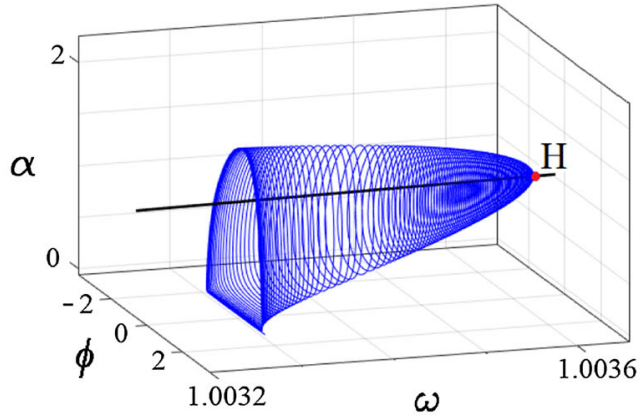

(a) $A=0.0075$

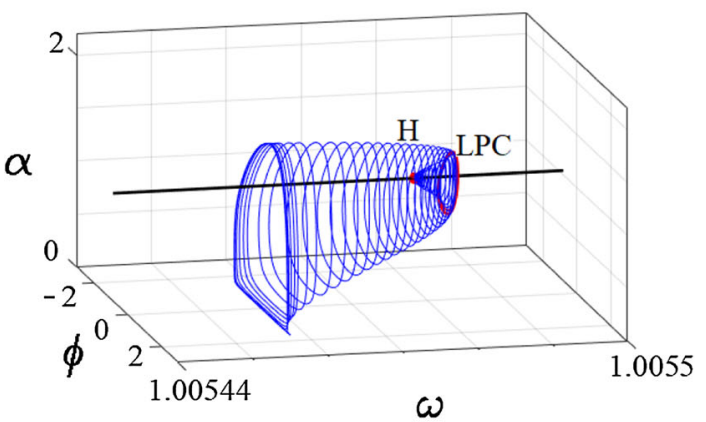

(b) $A=0.0070$

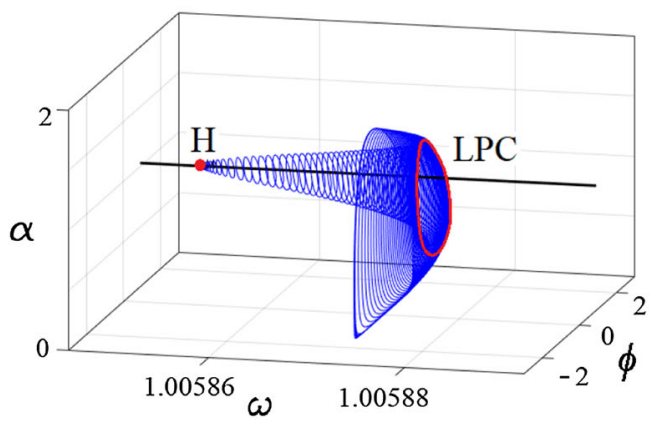

(c) $A=0.0065$

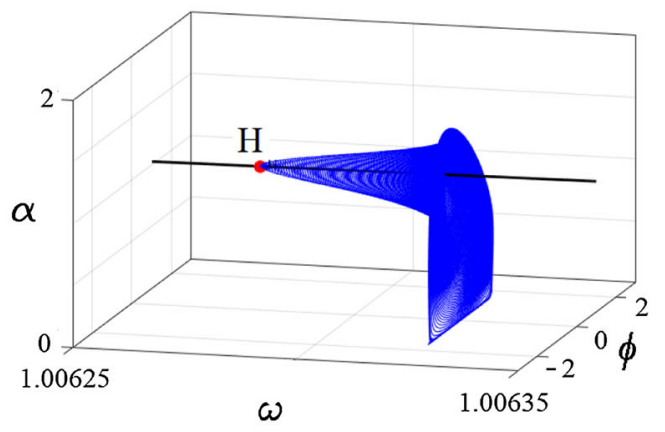

(d) $A=0.0060$

Fig. 14 The limit cycles before and after the General Hopf bifurcation point ( $H$ Hopf bifurcation point, $L P C$ Limit point bifurcation of cycles, the MatCont terminology for fold bifurcation of limit cycles) 


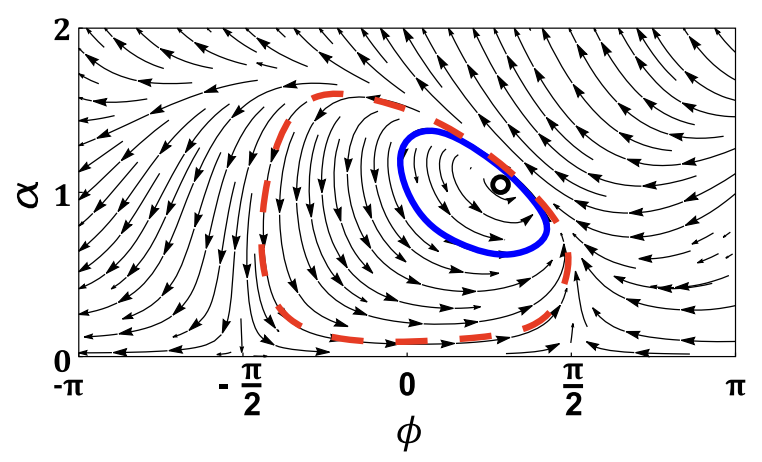

Fig. 15 Phase plane at $\omega=1.00587, A=0.0065$, with 2 coexisting limit cycles (solid line-stable, dashed line-unstable)

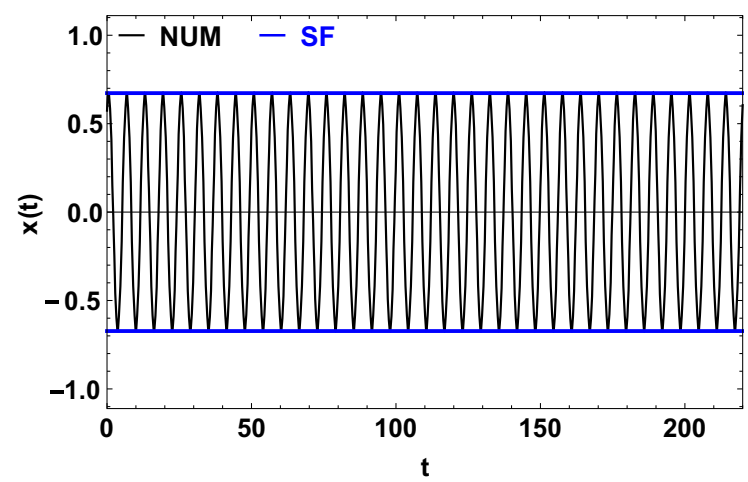

(a) Steady-state vibration.

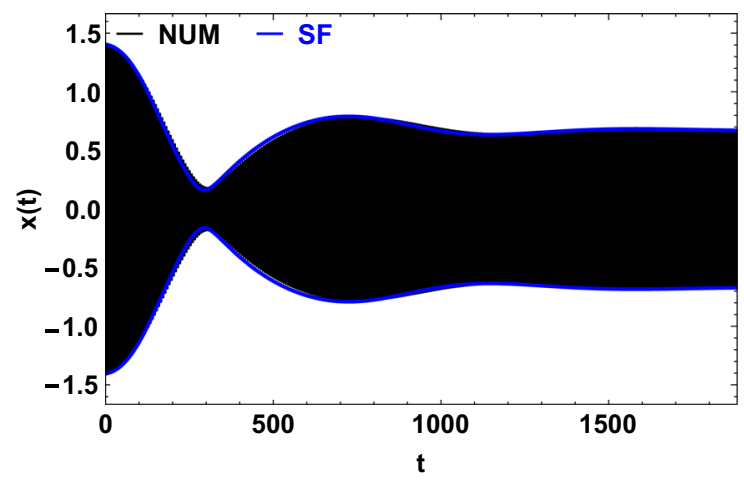

(b) Transition from transient response to steady-state vibration.

Fig. 16 Method of multiple scales solution and direct numerical solution $(A=0.01, \omega=1)$

presents the direct numerical solution (NUM) of Eq. (1) and the slow flow (SF) solution Eq. (19). Figure 17a demonstrates that initially the slow flow solution correctly approximates the envelope of the numerical solu-

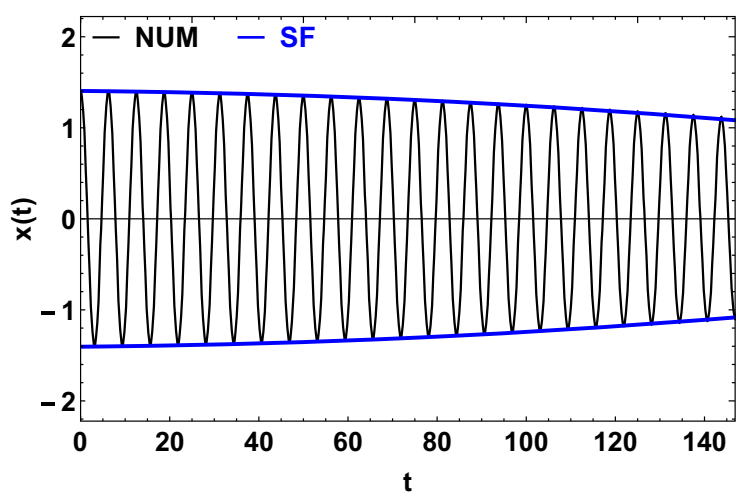

(a) $t \in[0,30 \tau]$

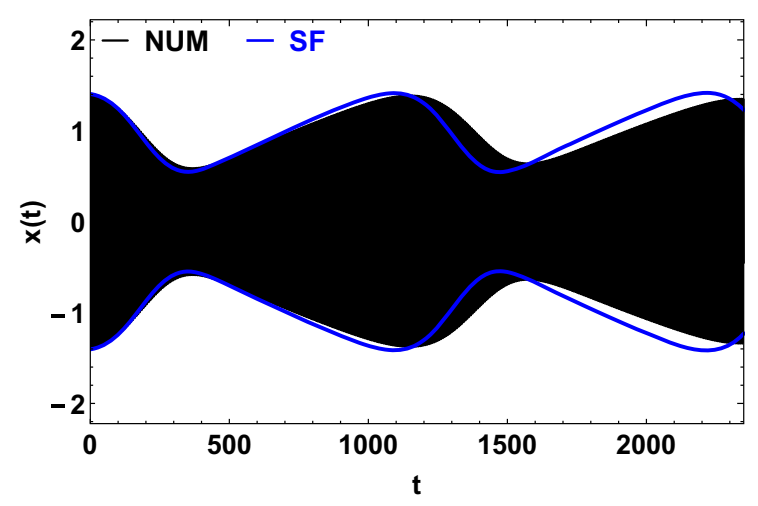

(b) $t \in[0,500 \tau]$

Fig. 17 Method of multiple scales solution and direct numerical solution $(A=0.01, \omega=1.0034)$ shown on different time intervals

tion, while Fig. 17b shows the long-term drift of the phase.

\subsection{Primary resonance}

The amplitude of the first harmonic (with frequency $\omega)$ of the numerical solution was determined in the time interval $\left[T_{1}, T_{2}\right]=[10 \tau, 50 \tau]$, with the following expression

$\alpha_{N U M}^{*}=\sqrt{\alpha_{\sin }^{2}+\alpha_{\cos }^{2}}$,

where

$\alpha_{\sin }=\frac{2}{T_{2}-T_{1}} \int_{T_{1}}^{T_{2}} x(t) \sin (\omega t) \mathrm{d} t$, 
Fig. 18 Primary resonance curve

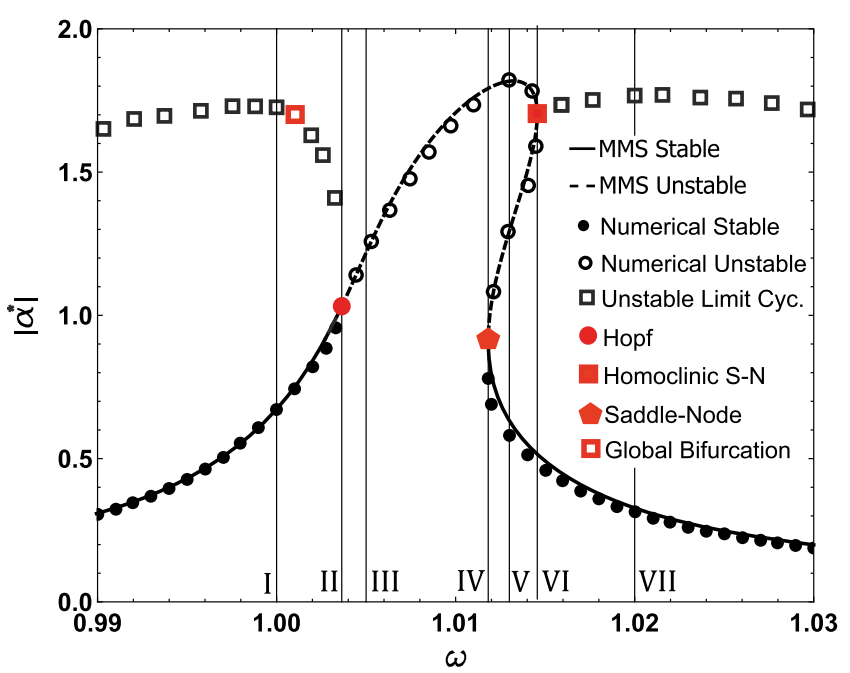

$\alpha_{\cos }=\frac{2}{T_{2}-T_{1}} \int_{T_{1}}^{T_{2}} x(t) \cos (\omega t) \mathrm{d} t$.

Figure 18 shows the amplitude $\alpha^{*}$ (Eq. 27) as a function of the forcing frequency $\omega$. The solid line indicates the stable multiple scales solution (MMS Stable); the dashed line shows the unstable multiple scales solution (MMS Unstable). The stable numerical equilibrium solutions are shown with filled circles; unstable numerical solution are depicted by empty circles. The solution was deemed unstable if its magnitude grew beyond a large number. The amplitude of the unstable limit cycles is depicted by empty squares (these amplitudes were determined using MatCont and bisection method). The thin vertical lines indicate the $\omega_{i}, i \in$ $\{I, I I, \ldots, V I I\}$ angular frequencies. The red markers correspond to the subcritical Hopf (Hopf), homoclinic saddle-node (Homoclinic S-N), global bifurcation of limit cycles (Global) and saddle-node (Saddle-Node) bifurcation points.

\section{Conclusions}

Equation (1) without forcing admits a subcritical Hopf bifurcation [18]. Forcing and Hopf bifurcation together may yield complicated motions [62]. Plaut and Hsieh [63] studied a forced 1-DOF mechanical system with delay and found periodic, chaotic and unbounded responses. Daqaq et al. investigated a harmonically forced delay system and got similar primary resonance curve [23]. The Taylor expansion of forced delay equation of machine tool vibration was investigated in [10].

Here we utilized the method of multiple scales to derive the slow flow equations for the forced delay equation Eq. (1). Equlibria of the slow flow were given by implicit algebraic equations. Analytical expressions were derived for the saddle-node and Hopf bifurcations of the slow flow equations. Bifurcation analysis of the slow flow equations has been shown for some numerical values of the parameters. Sub- and supercritical Hopf, generalized Hopf (Bautin), saddle-node, homoclinic saddle-node bifurcations were found. The bifurcations were illustrated with phase portraits and direct numerical integration of the original equation. Using MatCont, we determined the Generalized Hopf point of the system and located the local limit cycles. The analysis presented here demonstrates the rich dynamics of the system.

Acknowledgements Open access funding provided by Budapest University of Technology and Economics (BME). This project was supported by the New National Excellence Program (UNKP-18-2-I-BME-134) of the Ministry of Human Capacities of Hungary. The research reported in this paper was supported by the Higher Education Excellence Program of the Ministry of Human Capacities in the frame of Water Science \& Disaster Prevention research area of Budapest University of Technology and Economics (BME FIKP-VÍZ).

\section{Compliance with ethical standards}

Conflict of interest The authors declare that they have no conflict of interest. 
Open Access This article is distributed under the terms of the Creative Commons Attribution 4.0 International License (http:// creativecommons.org/licenses/by/4.0/), which permits unrestricted use, distribution, and reproduction in any medium, provided you give appropriate credit to the original author(s) and the source, provide a link to the Creative Commons license, and indicate if changes were made.

\section{References}

1. Balachandran, B., Kalmár-Nagy, T., Gilsinn, D.E.: Delay Differential Equations. Springer, Berlin (2009)

2. Erneux, T.: Applied Delay Differential Equations, vol. 3. Springer, Berlin (2009)

3. Smith, H.L.: An Introduction to Delay Differential Equations with Applications to the Life Sciences, vol. 57. Springer, New York (2011)

4. Breda, D., Maset, S., Vermiglio, R.: Stability of Linear Delay Differential Equations: A Numerical Approach with MATLAB. Springer, Berlin (2014)

5. Wahi, P., Chatterjee, A.: Regenerative tool chatter near a codimension 2 Hopf point using multiple scales. Nonlinear Dyn. 40(4), 323-338 (2005)

6. Nayfeh, A.H.: Order reduction of retarded nonlinear systems-the method of multiple scales versus centermanifold reduction. Nonlinear Dyn. 51(4), 483-500 (2008)

7. Oztepe, G.S., Choudhury, S.R., Bhatt, A.: Multiple scales and energy analysis of coupled Rayleigh-van der Pol oscillators with time-delayed displacement and velocity feedback: Hopf bifurcations and amplitude death. Far East J. Dyn. Syst. 26(1), 31 (2015)

8. Rahman, Z., Burton, T.: On higher order methods of multiple scales in non-linear oscillations-periodic steady state response. J. Sound Vib. 133(3), 369-379 (1989)

9. Wang, H., Hu, H.: Remarks on the perturbation methods in solving the second-order delay differential equations. Nonlinear Dyn. 33(4), 379-398 (2003)

10. Moradi, H., Bakhtiari-Nejad, F., Movahhedy, M.R., Ahmadian, M.T.: Nonlinear behaviour of the regenerative chatter in turning process with a worn tool: forced oscillation and stability analysis. Mech. Mach. Theory 45(8), 1050-1066 (2010)

11. Rand, R.H.: Lecture Notes on Nonlinear Vibrations (2012). https://ecommons.cornell.edu/handle/1813/28989

12. Pakdemirli, M., Karahan, M.M.F.: A new perturbation solution for systems with strong quadratic and cubic nonlinearities. Math. Methods Appl. Sci. 33(6), 704-712 (2010)

13. MacDonald, N.: Harmonic balance in delay-differential equations. J. Sound Vib. 186(4), 649-656 (1995)

14. Kalmár-Nagy, T.: Delay-differential models of cutting tool dynamics with nonlinear and mode-coupling effects. $\mathrm{PhD}$ thesis (2002)

15. Liu, L., Kalmár-Nagy, T.: High-dimensional harmonic balance analysis for second-order delay-differential equations. J. Vib. Control 16(7-8), 1189-1208 (2010)

16. Atay, F.M.: Van der Pol's oscillator under delayed feedback. J. Sound Vib. 218(2), 333-339 (1998)
17. Insperger, T., Stépán, G.: Semi-discretization for TimeDelay Systems: Stability and Engineering Applications, vol. 178. Springer, Berlin (2011)

18. Kalmár-Nagy, T., Stépán, G., Moon, F.C.: Subcritical Hopf bifurcation in the delay equation model for machine tool vibrations. Nonlinear Dyn. 26(2), 121-142 (2001)

19. Molnár, T.G., Dombóvári, Z., Insperger, T., Stépán, G.: On the analysis of the double Hopf bifurcation in machining processes via centre manifold reduction. Proc. R. Soc. A 473(2207), 20170502 (2017)

20. Faria, T., Magalhães, L.T.: Normal forms for retarded functional differential equations with parameters and applications to Hopf bifurcation. J. Differ. Equ. 122(2), 181-200 (1995)

21. Hu, H., Dowell, E.H., Virgin, L.N.: Resonances of a harmonically forced Duffing oscillator with time delay state feedback. Nonlinear Dyn. 15(4), 311-327 (1998)

22. Daqaq, M.F., Vogl, G.W.: Frequency Island in the Primary Resonance of Nonlinear Delay Systems. ENOC, Dubai (2008)

23. Daqaq, M.F., Alhazza, K.A., Qaroush, Y.: On primary resonances of weakly nonlinear delay systems with cubic nonlinearities. Nonlinear Dyn. 64(3), 253-277 (2011)

24. Shao, S., Masri, K., Younis, M.I.: The effect of time-delayed feedback controller on an electrically actuated resonator. Nonlinear Dyn. 74(1-2), 257-270 (2013)

25. Masri, K.M., Shao, S., Younis, M.I.: Delayed feedback controller for microelectromechanical systems resonators undergoing large motion. J. Vib. Control 21(13), 2604-2615 (2015)

26. Ji, J.: Secondary resonances of a quadratic nonlinear oscillator following two-to-one resonant hopf bifurcations. Nonlinear Dyn. 78(3), 2161-2184 (2014)

27. Ji, J.: Two families of super-harmonic resonances in a timedelayed nonlinear oscillator. J. Sound Vib. 349, 299-314 (2015)

28. Ji, J., Brown, T.: Periodic and chaotic motion of a timedelayed nonlinear system under two coexisting families of additive resonances. Int. J. Bifurc. Chaos 27(05), 1750066 (2017)

29. Ji, J., Zhou, J.: Coexistence of two families of sub-harmonic resonances in a time-delayed nonlinear system at different forcing frequencies. Mech. Syst. Signal Process. 93, 151163 (2017)

30. Hamdi, M., Belhaq, M.: Quasi-periodic oscillation envelopes and frequency locking in rapidly vibrated nonlinear systems with time delay. Nonlinear Dyn. 73(1-2), 1-15 (2013)

31. Grudziński, K., Kostek, R.: An analysis of nonlinear normal contact microvibrations excited by a harmonic force. Nonlinear Dyn. 50(4), 809-815 (2007)

32. Tobias, S., Fishwick, W.: Theory of regenerative machine tool chatter. Engineer 205(7), 199-203 (1958)

33. Tobias, S.: The vibrations of vertical milling machines under test and working conditions. Proc. Inst. Mech. Eng. 173(1), 474-510 (1959)

34. Tobias, S.A.: Machine-Tool Vibration. Wiley, Hoboken (1965)

35. Tlusty, J., Polacek, A., Danek, C., Spacek, J.: Selbsterregte Schwingungen an Werkzeugmaschinenen. VEB Verlag Technik, Berlin (1962) 
36. Stépán, G.: Retarded Dynamical Systems: Stability and Characteristic Functions. Longman Scientific \& Technical, Harlow (1989)

37. Stépán, G.: Delay-differential equation models for machine tool chatter. Dyn. Chaos Manuf. Process. 471152935, 165192 (1998)

38. Komanduri, R., Brown, R.: On the mechanics of chip segmentation in machining. J. Eng. Ind. 103(1), 33-51 (1981)

39. Burns, T.J., Davies, M.A.: Nonlinear dynamics model for chip segmentation in machining. Phys. Rev. Lett. 79(3), 447 (1997)

40. Gillespie, L.K., Blotter, P.: The formation and properties of machining burrs. J. Eng. Ind. 98(1), 66-74 (1976)

41. Vyas, A., Shaw, M.: Mechanics of saw-tooth chip formation in metal cutting. J. Manuf. Sci. Eng. 121(2), 163-172 (1999)

42. Zhang, B., Bagchi, A.: Finite element simulation of chip formation and comparison with machining experiment. J. Eng. Ind. 116(3), 289-297 (1994)

43. Obikawa, T., Sasahara, H., Shirakashi, T., Usui, E.: Application of computational machining method to discontinuous chip formation. J. Manuf. Sci. Eng. 119(4B), 667-674 (1997)

44. Wang, C., Xie, Y., Zheng, L., Qin, Z., Tang, D., Song, Y.: Research on the chip formation mechanism during the highspeed milling of hardened steel. Int. J. Mach. Tools Manuf. 79, 31-48 (2014)

45. Shaw, M., Vyas, A.: Chip formation in the machining of hardened steel. CIRP Ann. 42(1), 29-33 (1993)

46. Davies, M.A., Burns, T.J.: Thermomechanical oscillations in material flow during high-speed machining. Philos. Trans. R. Soc. Lond. Ser. A Math. Phys. Eng. Sci. 359(1781), 821846 (2001)

47. Csernák, G., Pálmai, Z.: Exploration of the chaotic phenomena induced by fast plastic deformation of metals. Int. J. Adv. Manuf. Technol. 40(3-4), 270-276 (2009)

48. Pálmai, Z., Csernák, G.: Chip formation as an oscillator during the turning process. J. Sound Vib. 326(3-5), 809-820 (2009)

49. Brehl, D., Dow, T.: Review of vibration-assisted machining. Precis. Eng. 32(3), 153-172 (2008)

50. Babitsky, V., Astashev, V.: Nonlinear dynamics and control of ultrasonically assisted machining. J. Vib. Control 13(5), 441-460 (2007)

51. Ostasevicius, V., Gaidys, R., Rimkeviciene, J., Dauksevicius, R.: An approach based on tool mode control for surface roughness reduction in high-frequency vibration cutting. J. Sound Vib. 329(23), 4866-4879 (2010)
52. Suzuki, N., Nakamura, A., Shamoto, E., Harada, K., Matsuo, M., Osada, M.: Ultraprecision micromachining of hardened steel by applying ultrasonic elliptical vibration cutting. In: Proceedings of 2003 International Symposium on Micromechatronics and Human Science MHS2003 (IEEE Cat. No. 03TH8717), pp. 221-226. IEEE (2003)

53. Hanna, N.H., Tobias, S.A.: A theory of nonlinear regenerative chatter. ASME J. Eng. Ind. 96(1974), 247-255 (1974)

54. Kuznetsov, Y.A.: Elements of Applied Bifurcation Theory, vol. 112. Springer, Berlin (2013)

55. Lelkes, J., Kalmár-Nagy, T.: Harmonically excited delay equation for machine tool vibrations. Paper No. DETC201886145. In: ASME IDET/CIE MSNDC (2018)

56. Lelkes, J., Kalmár-Nagy, T.: A nonlinear delay-differential equation with harmonic excitation. IFAC-PapersOnLine 51(14), 224-229 (2018)

57. Nekorkin, V.I.: Introduction to Nonlinear Oscillations. Wiley, Hoboken (2015)

58. Dhooge, A., Govaerts, W., Kuznetsov, Y.A., Meijer, H.G.E., Sautois, B.: New features of the software MatCont for bifurcation analysis of dynamical systems. Math. Comput. Model. Dyn. Syst. 14(2), 147-175 (2008)

59. Homburg, A.J., Sandstede, B.: Homoclinic and heteroclinic bifurcations in vector fields. Handb. Dyn. Syst. 3, 379-524 (2010)

60. Della Rossa, F., De Witte, V., Govaerts, W., Kuznetsov, Y.A.: Codimension 2 bifurcations of periodic orbits in matcont. http://www.staff.science.uu.nl/ kouzn101/NBA/ LCNF.pdf

61. Meijer, H.: Matcont tutorial: Ode gui version (2016)

62. Gambaudo, J.-M.: Perturbation of a Hopf bifurcation by an external time-periodic forcing. J. Differ. Equ. 57(2), 172199 (1985)

63. Plaut, R.H., Hsieh, J.-C.: Chaos in a mechanism with time delays under parametric and external excitation. J. Sound Vib. 114(1), 73-90 (1987)

Publisher's Note Springer Nature remains neutral with regard to jurisdictional claims in published maps and institutional affiliations. 